\title{
Odor generalization according to vibrational spectra
}

\author{
Klio Maniati ${ }^{1,2^{*}}$, Efthimios MC Skoulakis ${ }^{1}$, Luca Turin ${ }^{1}$ \\ From 1st International Workshop on Odor Spaces \\ Hannover, Germany. 4-7 September 2013
}

Much has been written and speculated about the potential activation mechanism of the olfactory receptors by an odorant molecule but the answer remains to this day unknown. Recently, there have been conclusive results in favor of the vibrational theory by means of deuterated odorants. These molecules, when compared to their hydrogen isotopes have unaltered shape but different vibrational profiles and, it turns out, a different odor. As shown by Franco et al. 2011, the ability of Drosophila melanogaster to avoid a deuterated compound when trained to avoid a nitrile and vice versa provides strong evidence for a vibrational component in olfaction. This phenomenon takes place because the C-D bond stretch vibration (deuterated compound) is at the same energy as the $\mathrm{C} \equiv \mathrm{N}$ bond stretch. The flies sensing the vibrations "confuse" one molecule for the other.

Having this very strong proof for the vibrational theory to build on, we continued our experimental strategy on the same mode, using Drosophila flies in T-maze, behavioral experiments. Indeed, Drosophila do not only generalize between the $\mathrm{C}$-D and the $\mathrm{C} \equiv \mathrm{N}$ bond but, also, as we show here, they generalize between the $\mathrm{S}-\mathrm{H}$ and the $\mathrm{B}-\mathrm{H}$ bond, both in the $2550 \mathrm{~cm}^{-1}$ region of the Infrared spectrum. A good pair of odorants to test the resemblance in smell between the B-H and the S-H bonds is decaborane and b-mercaptoethanol, two molecules totally dissimilar in shape and chemistry. This effect has never been investigated before, mainly because of the toxicity of boron compounds and their lack of use by experts in smell. The flies are shocked against one of the two odorants for 1 minute (12 shocks of $90 \mathrm{~V}$ at a 4 seconds interval) and are then, in the testing step, asked to chose between the other compound and air. They show a clear avoidance of the testing odorant.
A second way the topic is approached here is with the use of two isomers, which have different spectra. It is known that 2-hydroxynitriles [cyanohydrins] do not smell of nitriles. Remarkably, this fact has a ready vibrational explanation: the $\mathrm{C} \equiv \mathrm{N}$ stretch peak in the IR spectrum of cyanohydrins is absent or very small. It is shown here that flies, like us seem not to perceive the nitrile character in 2-hydroxybutyronitrile. In the contrary, as expected, the isomer 3-hydroxybutyronitrile [which has a $\mathrm{C} \equiv \mathrm{N}$ peak] is perceived as a nitrile odorant by the flies. They actually avoid the 3-hydroxybutyronitrile, but not the 2- hydroxy-butyronitrile when shocked against a different nitrile (citronellyl nitrile). Flies are, once again proving that a) two odorants with totally different shapes but peaks of comparable absorbance in the IR spectrum smell alike and b) two isomers that obviously have identical shape but dissimilar spectra smell differently. The results seem to clearly show that flies use a vibrational process to smell.

\section{Authors' details \\ 'Biomedical Sciences Research Centre "Alexander Fleming", Vari, Greece. ${ }^{2}$ School of Chemical Engineering, National Technical University of Athens,} Greece.

Published: 16 April 2014

doi:10.1186/2044-7248-3-S1-P9

Cite this article as: Maniati et al:: Odor generalization according to vibrational spectra. Flavour 2014 3(Suppl 1):P9. 